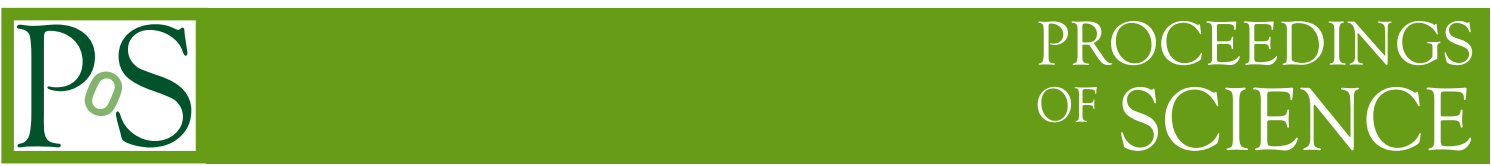

\title{
Hadron spectrum of QCD with one quark flavor
}

\author{
Federico Farchioni*, Gernot Münster, Tobias Sudmann*, Jaïr Wuilloud \\ Universität Münster, Institut für Theoretische Physik, \\ Wilhelm-Klemm-Strasse 9, D-48149 Münster, Germany \\ E-mail: farchion@mail.desy.de, tobias.sudmann@uni-muenster.de
}

\section{István Montvay}

Deutsches Elektronen-Synchrotron DESY, Notkestr. 85, D-22603 Hamburg, Germany

\section{Enno E. Scholz}

Physics Department, Brookhaven National Laboratory, Upton, NY 11973, USA

\begin{abstract}
The hadron spectrum of one flavor QCD is studied by Monte Carlo simulations. The Symanzik tree-level-improved Wilson action is used for the gauge field and the Wilson action for the fermion. The theory is simulated by a polynomial hybrid Monte Carlo algorithm (PHMC). The mass spectrum of hadronic bound states is investigated at two different lattice spacings: $a \simeq 0.37 r_{0}$ and $a \simeq 0.27 r_{0}$, corresponding to $\simeq 0.19 \mathrm{fm}$ and $\simeq 0.13 \mathrm{fm}$ in ordinary QCD. The lattice extension is fixed to $L \simeq 4.4 r_{0}(\simeq 2.2 \mathrm{fm})$. The lightest simulated quark mass corresponds to a pion with mass $\sim 270 \mathrm{MeV}$. Properties of the theory are analyzed by making use of the ideas of partially quenched chiral perturbation theory (PQChPT). The symmetry of the single flavor theory can be artificially enhanced by adding extra valence quarks, which can be interpreted as $u$ and $d$ quarks. Operators in the valence pion sector can be built. Masses and decay constants are analyzed by using PQChPT formulae at next-to-leading order.
\end{abstract}

The XXV International Symposium on Lattice Field Theory

July 30-4 August 2007

Regensburg, Germany

* Speaker. 


\section{Introduction and motivation}

QCD with one flavor of quarks $\left(N_{f}=1 \mathrm{QCD}\right)$ radically differs from QCD with two or more flavors due to the absence of a chiral symmetry: the abelian symmetry of the one flavor theory is washed out at the quantum level by the Adler-Bell-Jackiw anomaly. Only a vector symmetry survives, related to the conservation of the quark number. As a consequence of this, the main features of the phase structure and mass spectrum of the single flavor theory strongly deviate from the familiar picture, affected by the spontaneous breaking of the non-abelian chiral symmetry, of ordinary QCD. These unphysical features of $N_{f}=1 \mathrm{QCD}$ explain the little attention reserved to this kind of setup in past simulations (however with the exceptions of [1] and [2]).

This situation has changed in recent years, mainly due to the works of M. Creutz drawing the attention of the lattice community to open problems in the physical $\left(N_{f}>1\right)$ theory [3, 4]. Since these aspects are not directly related to the spontaneous breaking of the chiral symmetry, they find an equivalent in the single flavor theory. The latter represents therefore a simple setup for their investigation.

One question raised by Creutz [3], having a relevant phenomenological impact, is whether it is possible to define in an unambiguous way the case where one quark (say the $u$ quark) becomes massless. The arguments against an unique definition of the massless limit [3] essentially rest upon the $U(1)$ anomaly and should therefore hold a fortiori for the one flavor theory. A second aspect is the possibility of a spontaneous breaking of CP in QCD for special choices of the quark masses, conjectured for the first time by Dashen [5]. According to the Vafa-Witten theorem [6] a prerequisite for the spontaneous breaking of a discrete symmetry is a non positive fermion measure, which in $N_{f}=1$ QCD is possible for negative quark masses. The transition line is indeed expected to be located [7] on the negative real quark mass axis in the extended complex parameter space. In the case of the multi-flavor theory, the transition is excluded for physical values of the quark mass, but its nearby presence can nevertheless affect numerical simulations on the lattice [4]. So the main features of this transition are not of academic interest only.

Another intriguing aspect of one flavor QCD, emerging from string theory, is the connection with the $\mathscr{N}=1$ supersymmetric Yang-Mills theory (SYM). The equivalence of the two theories in the bosonic sector [8] can be proven at the planar level of a particular large $N_{c}$ limit (orientifold large $N_{c}$ limit) preserving balance between fermionic and bosonic degrees of freedom. Relics of SUSY are therefore expected in $N_{f}=1 \mathrm{QCD}$ (with $N_{c}=3$ ). A prediction of the orientifold equivalence [9], already studied in the literature [2], concerns in particular the size of the quark condensate.

Another important place where relics of SUSY in $N_{f}=1$ QCD can be investigated, considered in more detail in this contribution, is the low-lying bound-state spectrum [10]. In SYM the mass patterns are strongly constrained by SUSY. In particular low-energy models [11] predict a lowlying chiral supermultiplet including two scalar particles with opposite parity. ${ }^{1}$ In $N_{f}=1$ QCD these two particles can be easily identified with the $\eta$ and the $\sigma$ meson (the former picking up a mass through the anomaly). On the basis of the planar equivalence, their mass ratio including $O\left(1 / N_{c}\right)$ corrections is expected to be $m_{\sigma} / m_{\eta}=N_{c} /\left(N_{c}-2\right)$ [14].

\footnotetext{
${ }^{1}$ For recent lattice simulations of $\mathscr{N}=1 \mathrm{SYM}$, see $[12,13]$.
} 
The study of the mass spectrum of hadronic states requires reasonably large physical volumes, in order to be able to accommodate the bound-states in the finite box, and small (positive) quark masses. High statistics is required for a precise determination of the disconnected quark diagrams needed for the scalar meson masses, which are characterized by a high level of noise. High statistics is also important for the computation of the glueball masses. We apply here the Wilson lattice fermion action which has recently been shown $[15,16,17,18]$ to be well suited for such an investigation. Preliminary results with Stout-smeared links [19] in the Wilson fermion action will be also presented. Following [18] we apply in the gauge sector the tree level improved Symanzik action (tlSym).

The present exploratory study has been performed on $12^{3} \cdot 24$ and $16^{3} \cdot 32$ lattices with a lattice spacing corresponding in QCD units to $a \simeq 0.19 \mathrm{fm}$ and $a \simeq 0.13 \mathrm{fm}$, respectively. (We use the Sommer parameter [20] $r_{0}$ for setting the scale, fixed at the conventional value $r_{0} \equiv 0.5 \mathrm{fm}$.) For the future we plan to run simulations closer to the continuum limit.

As already mentioned, the sign of the quark determinant is an important issue in $N_{f}=1$ QCD (in particular, a negative determinant triggers the CP-violating phase transition). In the continuum, the fermion determinant is positive for positive quark mass. With Wilson lattice fermions for small quark masses, it can become negative due to quantum fluctuations. In most of our simulations the quark mass is large enough to prevent sign changes and the occurrence of a negative determinant is a rare event. For the lightest simulated quark masses however the sign of the quark determinant may potentially play a role and its impact in the hadron spectrum must be checked. In our simulations we could reach quite small quark masses down to $m_{q} \simeq 12 \mathrm{MeV}\left(m_{q} r_{0} \simeq 0.03\right)$, corresponding to a pion mass $m_{\pi} \simeq 270 \mathrm{MeV}$.

As we have argued in [10], it is useful to embed the $N_{f}=1$ QCD theory in a partially quenched theory with additional quark flavors. A particularly symmetric choice consists in taking the $\left(N_{V}\right)$ valence quark flavors degenerate with the sea quark: in this case the combined sea and valence sector is characterized by an exact $\mathrm{SU}\left(N_{V}+1\right)$ flavor symmetry. In this fictitious multi-flavor theory a PCAC quark mass can be naturally defined. We take this quantity as an operative definition of the quark mass for the (unitary) one-flavor theory. Also, a partially quenched chiral perturbation theory (PQChPT) can be set-up, exactly as in the $N_{f}>1$ case. The latter reduces to an effective theory of the $\eta$ meson in the unitary sector without valence quarks. The predictions of this PQChPT will be compared against our numerical data.

The plan of this contribution is as follows: in the next section the partially quenched viewpoint is introduced and PQChPT is considered for it. In Section 3 some information on the simulation algorithm and on the computation of the sign of the determinant are given. Section 4 is devoted to the presentation of our numerical results on the hadron spectrum, while Section 5 discusses the partially quenched data. The last section contains summary and outlook.

\section{Partially quenched QCD}

The symmetry of the one flavor theory can be artificially enhanced by adding extra valence quarks which are quenched, namely not taken into account in the Boltzmann-weight of the gauge configurations by their fermion determinants. A theoretical description of the resulting partially quenched theory can be obtained through the introduction of ghost quarks [21]. In this method the 
functional integral over the ghost quark fields $\tilde{\psi}$ cancels the fermion determinant of the valence quarks $\psi_{V}$,

$$
\begin{aligned}
& \int \mathscr{D} A \mathscr{D}\left[\psi_{S} \bar{\psi}_{S}\right] \mathscr{D}\left[\psi_{V} \bar{\psi}_{V}\right] \mathscr{D}[\tilde{\psi} \tilde{\tilde{\psi}}] \mathrm{e}^{-S_{\mathrm{g}}-\bar{\psi}_{S}\left(\gamma_{\mu} D_{\mu}+m_{S}\right) \psi_{S}-\bar{\psi}_{V}\left(\gamma_{\mu} D_{\mu}+m_{V}\right) \psi_{V}-\bar{\psi}\left(\gamma_{\mu} D_{\mu}+m_{V}\right) \tilde{\psi}} \\
= & \int \mathscr{D} A \mathrm{e}^{-S_{\mathrm{g}}} \frac{\operatorname{det}\left(\gamma_{\mu} D_{\mu}+m_{V}\right)}{\operatorname{det}\left(\gamma_{\mu} D_{\mu}+m_{V}\right)} \operatorname{det}\left(\gamma_{\mu} D_{\mu}+m_{S}\right),
\end{aligned}
$$

and only the determinant of the sea $(S)$ quark remains in the measure. In principle, one might consider any number of quenched valence quarks with any mass values. In our approach we take two valence quarks $u$ and $d$ with masses $m_{V}$ and one sea quark $s$ with mass $m_{S}$. For our purpose the case of degenerate valence and sea quark mass $m_{V}=m_{S}$ is particularly convenient (which is admittedly an unconventional kind of partially quenching). Observe that in this symmetric setup the exact number of valence quarks $N_{V}$ is immaterial, so our position $N_{F} \equiv N_{V}+N_{f}=2+1$ is just suggested by analogy with the case realized in nature. (Of course, in order to be able to build bound states containing two different quark flavors as mesons and nucleons, one needs $N_{V} \geq 1$.)

At the point of vanishing quark masses (see below) the generic partially quenched theory has a graded $\mathrm{SU}\left(N_{F} \mid N_{V}\right)_{L} \otimes \mathrm{SU}\left(N_{F} \mid N_{V}\right)_{R}$ symmetry, which is broken spontaneously into a "flavor" symmetry $\mathrm{SU}\left(N_{F} \mid N_{V}\right)$, also valid for non-vanishing degenerate quark masses. The $\mathrm{SU}\left(N_{F}\right)$ subgroup represents the flavor symmetry in the combined sea and valence quark sectors. The latter symmetry implies that the hadronic bound states appear in exactly degenerate $\mathrm{SU}\left(N_{F}\right)$ multiplets for $m_{V}=m_{S}$.

In particular, this extended theory contains a degenerate octet of pseudoscalar mesons ("pions" $\left.\pi^{a}, a=1, \ldots, 8\right)$ satisfying an $\mathrm{SU}(3)$-symmetric PCAC relation. Considering the divergence of the axial-vector current $A_{x \mu}^{a}$ and pseudoscalar density $P_{x}^{a}$ we can define the bare PCAC quark mass in lattice units as usual

$$
\operatorname{am}_{\mathrm{PCAC}} \equiv \frac{\left\langle\partial_{\mu}^{*} A_{x \mu}^{+} P_{y}^{-}\right\rangle}{2\left\langle P_{x}^{+} P_{y}^{-}\right\rangle} .
$$

Here the indices + and - refer to the "charged" components corresponding to $\lambda_{a} \pm \mathrm{i} \lambda_{b}$ (with $\lambda_{a, b}$ some off-diagonal Gell-Mann matrices) and $\partial_{\mu}^{*}$ denotes the backward lattice derivative. Due to the exact SU(3)-symmetry, the renormalized quark mass corresponding to $m_{\mathrm{PCAC}}$ can be defined by an $\mathrm{SU}(3)$-symmetric multiplicative renormalization:

$$
m_{\mathrm{PCAC}}^{\mathrm{R}}=\frac{Z_{A}}{Z_{P}} m_{\mathrm{PCAC}}
$$

As we will confirm numerically in sec. 5, the masses of the "pions" can be made to vanish by suitably tuning the bare quark mass on the lattice. In this situation the renormalized quark mass (2.3) vanishes, too. We stress here that the pions are not particles in the physical spectrum of the theory. Nevertheless their properties as mass and decay constant are well defined quantities which can be computed on the lattice. The same applies for the PCAC quark mass $m_{\mathrm{PCAC}}^{\mathrm{R}}$ which can be therefore regarded as a potential candidate for a definition of the quark mass in this theory.

\subsection{Chiral perturbation theory}

The dependence of pion properties upon the quark masses can be determined in partially quenched chiral perturbation theory (PQChPT) $[22,23]$. The effect of the finite lattice spacing 
$a$ can be also included $[24,25,26,27,28]$. The pseudo-Goldstone fields are parameterized by a graded matrix

$$
U(x)=\exp \left(\frac{\mathrm{i}}{F_{0}} \Phi(x)\right)
$$

which in our case is in the supergroup $\mathrm{SU}(3 \mid 2)$. (The normalization of $F_{0}$ is chosen such that its phenomenological value is $\simeq 86 \mathrm{MeV}$.) The commuting elements of the graded matrix $\Phi$ represent the pseudo-Goldstone bosons made from a quark and an anti-quark with equal statistics, while the anticommuting elements represent pseudo-Goldstone fermions which are built from one fermionic quark and one bosonic quark. The supertrace of $\Phi$ has to vanish, which can be implemented by a suitable choice of generators [29].

We have calculated both the masses and decay constants of the pseudo-Goldstone bosons in next-to-leading order of partially quenched chiral perturbation theory along the lines of Ref. [29], including $\mathscr{O}(a)$ lattice artifacts [26]. The quark masses enter the expressions in the combinations

$$
\chi_{V}=2 B_{0} m_{V}, \quad \chi_{S}=2 B_{0} m_{S}, \quad \chi_{\mathrm{PCAC}}=2 B_{0} m_{\mathrm{PCAC}}^{\mathrm{R}}
$$

with the usual leading order low-energy constant $B_{0}$; the lattice spacing enters in the combination

$$
\rho=2 W_{0} a
$$

where $W_{0}$ is another, lattice-specific, low-energy constant. We have calculated the masses of the pions and mixed mesons (degenerate in the special case $m_{V}=m_{S}$ ). The next-to-leading-order expression in terms of the (renormalized) PCAC quark mass is

$$
\begin{aligned}
m_{\pi}^{2}=\chi_{\mathrm{PCAC}}+\frac{\chi_{\mathrm{PCAC}}^{2}}{16 \pi^{2} F_{0}^{2}} \ln \frac{\chi_{\mathrm{PCAC}}}{\Lambda^{2}}+\frac{8}{F_{0}^{2}}\left[\left(2 L_{8}-L_{5}+2 L_{6}-L_{4}\right) \chi_{\mathrm{PCAC}}^{2}\right. \\
\left.+\left(W_{8}+W_{6}-W_{5}-W_{4}-2 L_{8}+L_{5}-2 L_{6}+L_{4}\right) \chi_{\mathrm{PCAC}} \rho\right]
\end{aligned}
$$

where the usual next-to-leading order low-energy parameters $L_{i}$ appear, together with additional ones $\left(W_{i}\right)$ describing lattice artifacts. For the decay constant we obtain in this case

$$
F_{\pi}=F_{0} \cdot\left\{1-\frac{\chi_{\mathrm{PCAC}}}{32 \pi^{2} F_{0}^{2}} \ln \frac{\chi_{\mathrm{PCAC}}}{\Lambda^{2}}+\frac{8}{F_{0}^{2}}\left[\left(L_{5}+L_{4}\right) \chi_{\mathrm{PCAC}}+\left(W_{5}+W_{4}-L_{5}-L_{4}\right) \rho\right]\right\} .
$$

Observe that as expected the results are independent of $N_{V}$. In particular, calculating the quantities in this model with $N_{V}=1$, which corresponds to a representation the supergroup $\mathrm{SU}(2 \mid 1)$, reproduces (2.7) and (2.8).

The analysis can be extended by relating the pion mass to the mass of the "physical" $\eta$. The inclusion of the singlet can be achieved by relaxing the constraint of a vanishing supertrace [22, 29], and associating it with the field

$$
\Phi_{0}(x)=\operatorname{sTr} \Phi(x) .
$$

The effective Lagrangian then contains additional terms depending on $\Phi_{0}$ :

$$
\Delta \mathscr{L}=\alpha \partial_{\mu} \Phi_{0} \partial_{\mu} \Phi_{0}+m_{\Phi}^{2} \Phi_{0}^{2}+\mathscr{O}\left(\Phi_{0}^{3}\right)
$$


where $\alpha$ and $m_{\Phi}$ are free parameters in this context. We will use in the following the leading order expression for the mass of the $\eta$, which reads

$$
m_{\eta}^{2}=\frac{m_{\Phi}^{2}+\chi_{\mathrm{PCAC}}}{1+\alpha}
$$

Our numerical results for $m_{\eta}$ allow to determine $\alpha$ and $m_{\Phi}$ (see Section 5).

\section{Simulation}

For the SU(3) gauge sector we apply the tree-level improved Symanzik (tlSym) action [30] including planar rectangular $(1 \times 2)$ Wilson loops:

$$
S_{g}=\beta \sum_{x}\left(c_{0} \sum_{\mu<v ; \mu, v=1}^{4}\left\{1-\frac{1}{3} \operatorname{Re} U_{x \mu \nu}^{1 \times 1}\right\}+c_{1} \sum_{\mu \neq v ; \mu, v=1}^{4}\left\{1-\frac{1}{3} \operatorname{Re} U_{x \mu \nu}^{1 \times 2}\right\}\right),
$$

with $c_{1}=-1 / 12$ and normalization condition $c_{0}=1-8 c_{1}$. The fermionic part of the lattice action is the simple (unimproved) Wilson action. With the goal of improving the stability of the Monte Carlo evolution at small quark masses, we also started simulations with Stout-smeared links [19] in the hopping matrix (see below).

The update algorithm is a Polynomial Hybrid Monte Carlo algorithm (PHMC) [31, 32] allowing the simulation of an odd number of fermion species. The present version [33] is based on a two-step polynomial approximation of the inverse fermion matrix with stochastic correction in the update chain: a sequence of PHMC trajectories is followed by a Metropolis accept-reject step with a higher precision polynomial. The polynomial approximation scheme and the stochastic correction in the update chain are taken over from the two-step multi-boson algorithm of Ref. [34]. A correction factor $C[U]$ in the measurement is associated with configurations for which eigenvalues of the (squared Hermitian) fermion matrix $Q^{2}[U]$ lie outside the validity interval of the polynomial approximation. We refer to [10] for more details on the algorithmic setup.

As mentioned in the Introduction, the sign $\sigma[U]$ of the fermion determinant $\operatorname{det} Q[U]$ has also to be included in the reweighting of the configurations. The expectation value of a quantity $A$ is therefore given by

$$
\langle A\rangle=\frac{\int[d U] \sigma[U] C[U] A[U]}{\int[d U] \sigma[U] C[U]} .
$$

For the computation of the sign $\sigma[U]$ we applied two methods. In the first we studied the spectral flow of the Hermitian fermion matrix [35]. For the $\kappa$-dependent computation of the low-lying eigenvalues of the Hermitian fermion matrix $Q[U]$ we followed in this case Ref. [36]. Alternatively, we computed the (complex) spectrum of the non-Hermitian matrix concentrating on the lowest real eigenvalues: sign changes are signaled by negative real eigenvalues. We applied the ARPACK Arnoldi routines [37] on a transformed Dirac operator. The (polynomial) transformation was tuned such that the real eigenvalues were projected outside the ellipsoidal bulk containing the whole eigenvalue spectrum [38]. This allows for an efficient computation of the real eigenvalues [39]. This latter method, on which we will rely in the future, delivers unambiguous results and can be simply automatized. 
Table 1: Summary of the runs: $12^{3} \cdot 24$ and $16^{3} \cdot 32$ lattices have lowercase and uppercase labels, respectively. The bar indicates runs with Stout-link in the fermion action (see text).

\begin{tabular}{c|c|c|c|c|c|c} 
& $\beta$ & $\kappa$ & $N_{\text {conf }}$ & plaquette & $\tau_{\text {plaq }}$ & $r_{0} / a$ \\
\hline \hline$a$ & 3.80 & 0.1700 & 5424 & $0.546041(66)$ & 12.5 & $2.66(4)$ \\
\hline$b$ & 3.80 & 0.1705 & 3403 & $0.546881(46)$ & 4.6 & $2.67(5)$ \\
\hline$c$ & 3.80 & 0.1710 & 2884 & $0.547840(67)$ & 7.6 & $2.69(5)$ \\
\hline \hline$A$ & 4.00 & 0.1600 & 1201 & $0.581427(36)$ & 4.3 & $3.56(5)$ \\
\hline$B$ & 4.00 & 0.1610 & 1035 & $0.582273(36)$ & 4.1 & $3.61(5)$ \\
\hline$C$ & 4.00 & 0.1615 & 1005 & $0.582781(32)$ & 3.3 & $3.73(5)$ \\
\hline \hline $\bar{A}$ & 4.00 & 0.1440 & 5600 & $0.577978(23)$ & 9.7 & $3.74(3)$ \\
\hline $\bar{B}$ & 4.00 & 0.1443 & 5700 & $0.578167(28)$ & 11.3 & $3.83(5)$ \\
\hline \hline
\end{tabular}

\subsection{Simulation details}

We performed simulations on a $12^{3} \cdot 24$ lattice with $\beta=3.8$ and on a $16^{3} \cdot 32$ with $\beta=4.0$. Information regarding the generated sets of configurations are reported in Table 1.

The sequences consisted of 3-6 PHMC individual trajectories. The precision of the first step of polynomial approximations was tuned such that the acceptance of the PHMC trajectories was about $0.80-0.85$. The same acceptance was required for the Metropolis test by tuning the total length of the trajectory (1.5-1.8). This resulted in a relatively high total acceptance of $0.64-0.72$. Optimization of the parameters of PHMC turned out to have a substantial impact on the integrated autocorrelation times of the average plaquette.

In the case of a Stout-link we consider one step of isotropic smearing with $\rho_{\mu v}=\rho=0.15$, $\mu, v=1, \ldots, 4$. The Stout-smearing has in general the beneficial effect, compared to the unsmeared action, of reducing the fluctuations of the smallest eigenvalue of the (squared) hermitian matrix, with the result that less exceptional configurations are observed. This allowed us to obtain smooth simulations down to quite small pion masses $m_{\pi} \simeq 270 \mathrm{MeV}$.

Taking the values of $r_{0} / a$ at the highest $\kappa$ 's for the runs at $\beta=3.8$ and $\beta=4.0$ and fixing $r_{0}=0.5 \mathrm{fm}$ by definition we obtain $a=0.186 \mathrm{fm}$ and $a=0.134 \mathrm{fm}$, respectively. The extensions of the $12^{3}$ and $16^{3}$ lattices are roughly constant: $L=2.23 \mathrm{fm}$ and $L=2.14 \mathrm{fm}$. (The Stout-smearing leaves $r_{0} / a$ essentially unchanged.)

For runs $b, c, \bar{A}$ and $\bar{B}$ there are cases where the eigenvalues of the fermion matrix are outside the approximation interval $[\varepsilon, \lambda]$ and therefore $C[U] \neq 1$. In run $c$ in particular there are 167 of such configuration out of 2884,26 of them with negative sign. However, even in this case the average value $\sigma[U] C[U]$ is very near to one: 0.9842 . The effect of the correction factors turns out to be quite weak in the case of the average plaquette and of $r_{0} / a$ : the effect on the average value of $r_{0} / a$ is only in the fifth digit (whereas the statistical error is in the third digit). This is not the case for low energy quantities as the low-lying hadron masses (see in the following). 
Table 2: Results for light hadron masses in $N_{f}=1 \mathrm{QCD}$ (the result with the asterisk has been obtained with higher statistics: 4900). Note that the glueball masses were obtained at small time separations and hence could be overestimated (see also text).

\begin{tabular}{c|c|c|c|c} 
& $a m_{\eta}$ & $a m_{\sigma}$ & $a m_{0^{++}}$ & $a m_{\Delta}$ \\
\hline \hline$a$ & $0.462(13)$ & $0.660(39)$ & $0.777(11)$ & $1.215(20)$ \\
\hline$b$ & $0.403(11)$ & $0.629(29)$ & $0.685(10)$ & $1.116(38)$ \\
\hline$c$ & $0.398(28)$ & $0.584(55)$ & $0.842(16)$ & $1.204(57)$ \\
\hline \hline$A$ & $0.455(17)$ & $0.607(57)$ & $1.083(79)$ & $1.006(15)$ \\
\hline$B$ & $0.380(18)$ & $0.554(52)$ & $1.032(66)$ & $0.960(15)$ \\
\hline$C$ & $0.316(22)$ & $0.613(67)$ & $0.943(41)^{*}$ & $0.876(26)$ \\
\hline \hline
\end{tabular}

\section{Hadron spectrum}

\subsection{Mesons}

For the meson states we consider the simplest interpolating operators in the pseudoscalar and scalar sectors:

$$
\begin{array}{ll}
\eta\left(0^{-}\right): & P(x)=\bar{\psi}(x) \gamma_{5} \psi(x), \\
\sigma\left(0^{+}\right): & S(x)=\bar{\psi}(x) \psi(x) .
\end{array}
$$

Corresponding states in the QCD spectrum are the $\eta^{\prime}(958)$ and $f_{0}(600)$ (or $\sigma$ ). In the case of the pseudoscalar mesons, invariance under the flavor group plays a special role when comparing with QCD states because of the U(1) axial anomaly.

The disconnected diagrams of the hadron correlators of $\eta$ and $\sigma$ were computed by applying stochastic sources with complex $Z_{2}$ noise and spin dilution. The method was already applied to the case of lattice SYM [13]. In order to optimize the computational load, also considering autocorrelations, we analyzed typically every fifth configuration, with 20 stochastic estimates each. The resulting statistics is $400-600$ on the smaller lattice and $\sim 200$ on the larger one.

\subsection{Baryons}

The simplest interpolating field in the baryon sector containing just one quark field is

$$
\Delta_{i}(x)=\varepsilon_{a b c}\left[\psi_{a}(x)^{T} C \gamma_{i} \psi_{b}(x)\right] \psi_{c}(x) .
$$

The low lying hadron state interpolated by the above operator is expected to have spin $3 / 2$ and positive parity $\left(\frac{3}{2}^{+}\right)$. This corresponds to the $\Delta^{++}(1232)$ of QCD if our dynamical fermion is interpreted as an $u$ quark (the $\Omega^{-}$baryon is more appropriate for larger quark masses).

A difficulty arises since the Rarita-Schwinger spinor (4.3) also contains a spin 1/2 component. We extract the wanted spin $3 / 2$ component by projection [40]:

$$
G_{3 / 2}(t)=\frac{1}{6} \operatorname{Tr}\left[G_{j i}(t) \gamma_{j} \gamma_{i}+G_{i i}(t)\right], \quad G_{j i}(t)=\sum_{\vec{x}}\left\langle\Delta_{j}(\vec{x}, t) \bar{\Delta}_{i}(0)\right\rangle .
$$




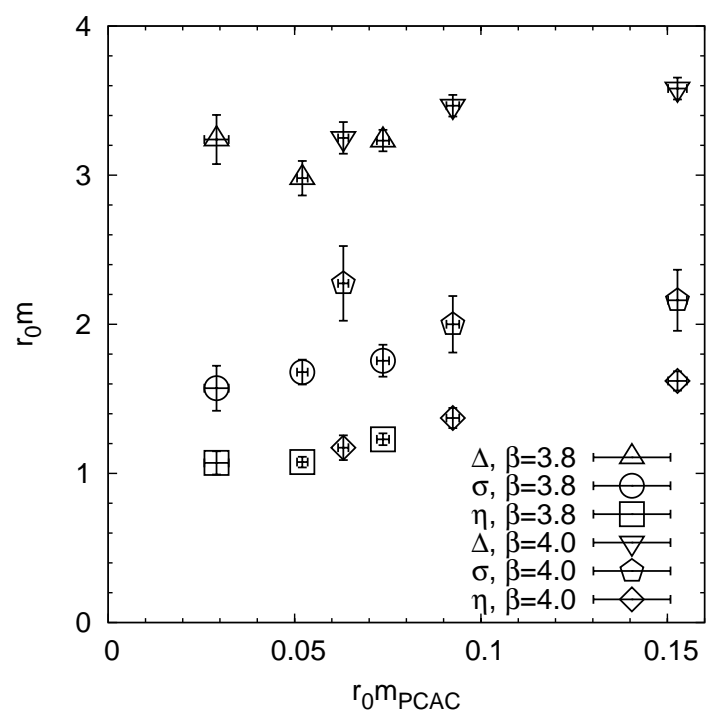

Figure 1: The mass of the lightest physical particles in one-flavor QCD as a function of the bare PCAC quark mass. The masses are multiplied by the scale parameter $r_{0}$ in order to obtain dimensionless quantities.

Since the baryon correlator does not contain disconnected diagrams, our full statistics could be taken for the computation of the masses in this case, namely 3000-4000 on the smaller lattice and $\sim 1000$ on the larger one.

\subsection{Glueballs}

Spin 0 states are also projected by purely gluonic operators. These are the glueballs, a well known object of investigation in lattice QCD. In particular the $0^{++}$glueball has the same quantum numbers as the $\sigma$ meson. In this first investigation we neglect possible mixings between the two states and consider only diagonal correlators.

We used the single spatial plaquette to obtain the mass of the $0^{++}$ground state. To increase the overlap of the operator with this state we performed APE smearing [41] and also applied variational methods [42] to obtain optimal glueball operators from linear combinations of the basic operators.

\subsection{Results}

The results for the hadron masses (only available for the runs without Stout-smearing) are reported in lattice units in Table 2. In Fig. 1 the hadron masses are plotted as a function of the bare PCAC quark mass $m_{\text {PCAC }}(2.3)$ defined in the partially quenched picture. Since we use physical units here, results from the two lattice spacings can be compared. The scaling is satisfactory for the case of $\eta$, whose mass could be computed with the best accuracy. The determination of the $\sigma$ meson mass seems to require large statistics.

The effect of the sign of the determinant in the hadron spectrum was investigated by computing the masses with or without the inclusion of the sign factor in the reweighting procedure. Only in the case of our run at the lightest quark mass, run $c$, a sizeable effect can be observed: here the sign of the determinant pushes up the masses by $7-10 \%$. 
Table 3: The PCAC quark mass $m_{\mathrm{PCAC}}$, the pion mass $m_{\pi}$ and non-renormalized decay constant $f_{\pi}$, and the nucleon mass $m_{N}$ in lattice units (only for the runs without Stout-smearing).

\begin{tabular}{c|l|l|l|l} 
& \multicolumn{1}{|c|}{$a m_{\mathrm{PCAC}}$} & \multicolumn{1}{|c}{$a m_{\pi}$} & \multicolumn{1}{|c}{$a f_{\pi}$} & \multicolumn{1}{c}{$a m_{N}$} \\
\hline \hline$a$ & $0.02771(45)$ & $0.3908(24)$ & $0.1838(11)$ & $1.0439(54)$ \\
\hline$b$ & $0.01951(39)$ & $0.3292(25)$ & $0.1730(15)$ & $0.956(27)$ \\
\hline$c$ & $0.0108(12)$ & $0.253(10)$ & $0.156(10)$ & $1.011(51)$ \\
\hline \hline$A$ & $0.04290(36)$ & $0.4132(21)$ & $0.1449(9)$ & $0.9018(44)$ \\
\hline$B$ & $0.02561(31)$ & $0.3199(22)$ & $0.1289(10)$ & $0.7978(53)$ \\
\hline$C$ & $0.01700(30)$ & $0.2635(24)$ & $0.1188(12)$ & $0.734(10)$ \\
\hline $\bar{A}$ & $0.01532(34)$ & $0.2316(49)$ & $0.09747(15)$ & \\
\hline $\bar{B}$ & $0.00886(75)$ & $0.1994(74)$ & $0.0852(49)$ & \\
\hline \hline
\end{tabular}

We observe that our statistics is not large enough to obtain an accurate estimate of the glueball masses. In particular, the results reported in Table 2 could be overestimated. Indeed, due to the high level of noise, large time-separations could not be included in the determinations; it is therefore possible that the latter are contaminated by excited states. In order to enhance the statistics we decided to store the gauge configuration more frequently (as was already applied for the continuation of run $C$ ).

\section{Partially quenched analysis}

The results for the partially quenched sector are collected in Table 3 and shown in Figure 2. This also includes the nucleon mass (only for the runs without Stout-smearing).

The partially quenched ChPT formulae are used to extract the corresponding low-energy coefficients from the pion data. Considering the number of lattice data at our disposal, a full fit including all the terms in the ChPT formulae is not possible, so we take only the continuum terms into account. We fitted the data for both $\beta$ values simultaneously neglecting the dependence of the renormalizations factors $Z_{A}$ and $Z_{P}$ upon the lattice coupling constant. Introducing the one-flavor low-energy constants

$$
\begin{aligned}
& \Lambda_{3}=4 \pi F_{0} \exp \left\{64 \pi^{2}\left(L_{4}+L_{5}-2 L_{6}-2 L_{8}\right)\right\} \\
& \Lambda_{4}=4 \pi F_{0} \exp \left\{64 \pi^{2}\left(L_{4}+L_{5}\right)\right\}
\end{aligned}
$$

the fit formulae of the renormalized values reduce to

$$
\begin{aligned}
m_{\pi}^{2} & =\chi_{\mathrm{PCAC}}+\frac{\chi_{\mathrm{PCAC}}^{2}}{16 \pi^{2} F_{0}^{2}} \ln \frac{\chi_{\mathrm{PCAC}}}{\Lambda_{3}^{2}}, \\
\frac{f_{\pi}^{\mathrm{R}}}{F_{0} \sqrt{2}} & =1-\frac{\chi_{\mathrm{PCAC}}}{32 \pi^{2} F_{0}^{2}} \ln \frac{\chi_{\mathrm{PCAC}}}{\Lambda_{4}^{2}} .
\end{aligned}
$$




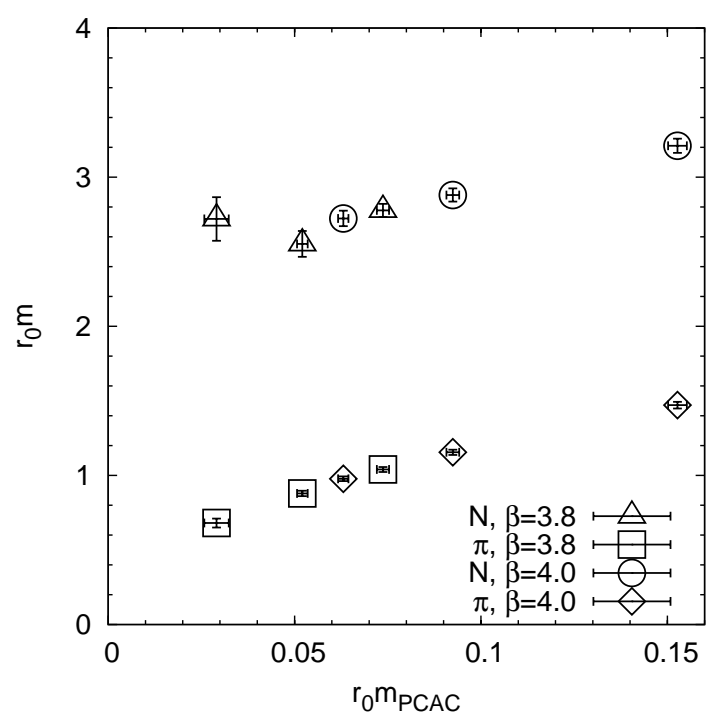

Figure 2: The mass of the valence pion and nucleon as a function of the bare PCAC quark mass.

The data and the fitted curves are shown in Fig. 3.

In order to improve the numerical results for the universal low-energy constants $\Lambda_{3,4}$, which do not explicitly depend on the lattice spacing $a$, we also performed fits to the ratios $[43,15]$

$$
\frac{m_{\pi}^{2}}{m_{\pi, \mathrm{ref}}^{2}}, \frac{f_{\pi}}{f_{\pi, \mathrm{ref}}}
$$

For this calculation we restricted ourself to the data at $\beta=4.0$ with reference point at $\kappa=0.1615$. We obtain in this case the results

$$
\begin{aligned}
& \frac{\Lambda_{3}}{F_{0}}=10.0 \pm 2.6, \\
& \frac{\Lambda_{4}}{F_{0}}=31.5 \pm 14.3,
\end{aligned}
$$

which, interestingly, are compatible with phenomenological values obtained from ordinary QCD [44]. The errors are however quite large (we hope to improve these determinations in the future).

In addition, we investigated the relation between the mass of the pion and of the physical $\eta$, reducing to formula (2.11) at leading-order. For this purpose we fitted simultaneously $m_{\pi}^{2}$ and $m_{\eta}^{2}$ as a function of the PCAC quark mass, again considering only $\beta=4.0$. This yields to

$$
\alpha=-0.03(19), \quad a m_{\Phi}=0.18(8),
$$

suggesting a vanishing $\alpha$. Using the value of $r_{0} / a$ extrapolated to vanishing PCAC quark mass and setting $\alpha=0$ we find

$$
a m_{\Phi}=0.19(2) \quad \text { or } \quad r_{0} m_{\Phi}=0.72(10)
$$

which means

$$
m_{\Phi}=284 \pm 40 \mathrm{MeV}
$$



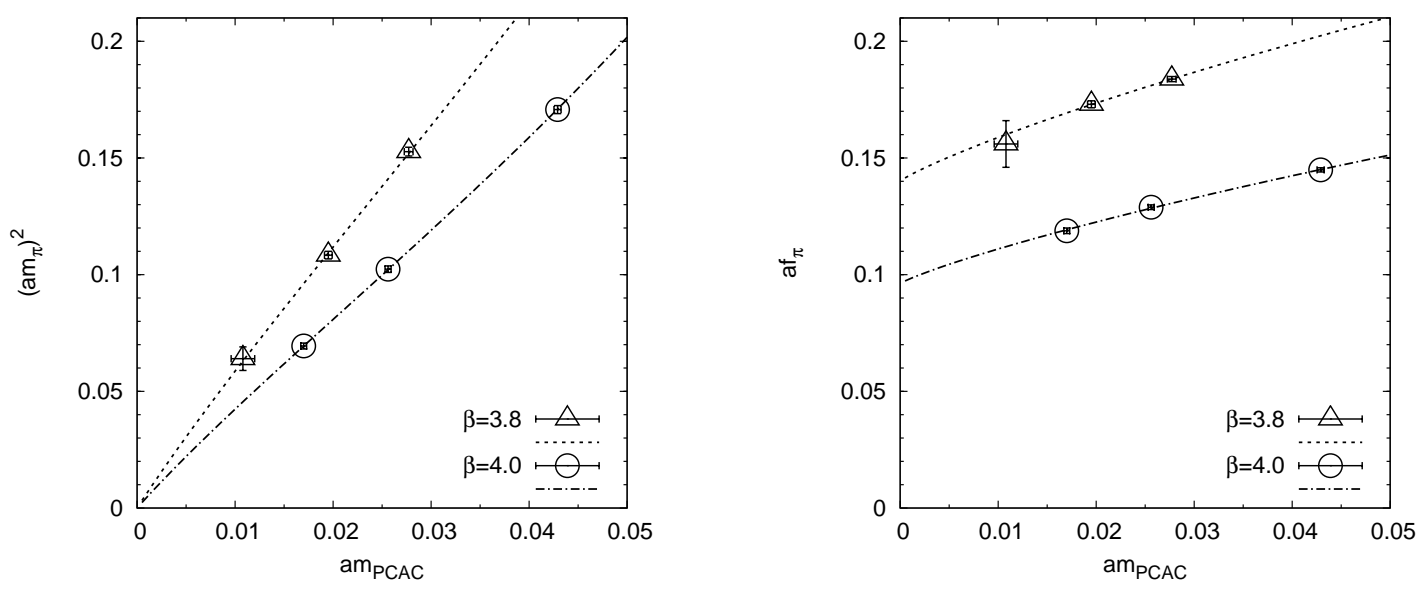

Figure 3: Pion masses squared and pion decay constants in lattice units and the results of the PQChPT fit.

in physical units.

The value of $m_{\Phi}$ can also be obtained from the Witten-Veneziano formula [45]

$$
m_{\Phi}^{2}=\frac{4 N_{f}}{\left(f_{\pi}^{\mathrm{R}}\right)^{2}} \chi_{t}
$$

valid at leading-order in the ('t Hooft) large $N_{c}$ limit. An estimate of the quenched topological susceptibility present in the literature is $\chi_{t}=(193 \pm 9 \mathrm{MeV})^{4}$ [46]. Using our value for $f_{\pi}^{R}$, which is subject to a sizeable statistical error, one would obtain $m_{\Phi}=450 \pm 170 \mathrm{MeV}$.

\section{Summary and outlook}

This first Monte Carlo investigation of $N_{f}=1$ QCD reveals the qualitative features of the low lying hadron spectrum of this theory. The lightest hadron is the pseudoscalar $\eta$ meson (see Table 2 and Figure 1) while the scalar meson, the $\sigma$, is about a factor 1.5 heavier. It is interesting to compare our data with the estimate in [47] $m_{\sigma} / m_{\eta} \simeq N_{c} /\left(N_{c}-2\right)=3$ for $N_{c}=3$. The above prediction applies for the massless theory and one could expect the agreement to improve for smaller quark masses. Our bare quark masses (estimated from the PCAC quark mass in the valence analysis) range between $10 \mathrm{MeV}$ and $60 \mathrm{MeV}$, while the lightest pion mass is $\sim 270 \mathrm{MeV}$.

The lightest baryon, the $\Delta\left(\frac{3}{2}^{+}\right)$, is by about a factor 3 heavier than the $\eta$ meson. The lightest scalar mass obtained with a glueball $0^{++}$operator lies between the $\sigma$ meson and the $\Delta$ baryon mass. However, this mass could be overestimated, since, due the high level of noise, only small time-separations could be included in the analysis.

In general, the mass measurements have relatively large errors between 3-10\%. In order to obtain more quantitative results, larger statistics and smaller quark masses are required. We hope to be able to make progresses in both directions [48] with our new simulations using Stout-smeared links in the fermion action. Some preliminary results were already presented in this contribution (see [49] for a test of this formulation in twisted mass QCD with $N_{f}=2$ ). 
The introduction of a partially quenched extension of the single flavor theory with valence quarks allows to define the bare quark mass in terms of the PCAC quark mass of the fictitious multi-flavor theory. The computation of the bare quark mass is intricate in the unitary theory due to the absence of a chiral symmetry (the arguments of [3] regard the definition of a renormalized quark mass). Comparison of lattice data with partially quenched chiral perturbation theory allowed the determination of some of the low-energy constants of the chiral Lagrangian. The latter are compatible, even if with large error, with recent lattice determinations for $N_{f}=2$ QCD.

A further direction of investigation for the future [48] is the CP-violating phase transition expected at negative quark masses [7]. For this aspect of the single flavor theory the non-positivity of the fermion measure plays an essential role.

\section{References}

[1] C. Alexandrou, A. Borici, A. Feo, Ph. de Forcrand, A. Galli, F. Jegerlehner and T. Takaishi, Phys. Rev. D 60 (1999) 034504; hep-lat/9811028.

[2] T. DeGrand, R. Hoffmann, S. Schaefer and Z. Liu, Phys. Rev. D 74 (2006) 054501; hep-th/0605147.

[3] M. Creutz, Phys. Rev. Lett. 92 (2004) 162003; hep-ph/0312225.

[4] M. Creutz, Phys. Rev. Lett. 92 (2004) 201601; hep-lat/0312018.

[5] R.F. Dashen, Phys. Rev. D 3 (1971) 1879.

[6] C. Vafa and E. Witten, Nucl. Phys. B 234 (1984) 173.

[7] M. Creutz, Phys. Rev. D 52, 2951 (1995); hep-th/9505112.

[8] A. Armoni, M. Shifman and G. Veneziano, Nucl. Phys. B 667 (2003) 170; hep-th/0302163; A. Armoni and M. Shifman, Nucl. Phys. B 670 (2003) 148; hep-th/0303109; A. Armoni, M. Shifman and G. Veneziano, Phys. Rev. Lett. 91 (2003) 191601; hep-th/0307097.

[9] A. Armoni, M. Shifman and G. Veneziano, Phys. Lett. B 579 (2004) 384; hep-th/0309013.

[10] F. Farchioni, I. Montvay, G. Münster, E. E. Scholz, T. Sudmann and J. Wuilloud, Eur. Phys. J. C 52, 305 (2007); arXiv:0706.1131 [hep-lat].

[11] G. Veneziano and S. Yankielowicz, Phys. Lett. B 113 (1982) 231.

[12] F. Farchioni, A. Feo, T. Galla, C. Gebert, R. Kirchner, I. Montvay, G. Münster and A. Vladikas [DESY-Münster-Roma Collaboration], Eur. Phys. J. C 23 (2002) 719; hep-lat/0111008.

[13] F. Farchioni and R. Peetz, Eur. Phys. J. C 39 (2005) 87; hep-lat/0407036.

[14] A. Armoni and E. Imeroni, Phys. Lett. B 631 (2005) 192; hep-th/0508107.

[15] F. Farchioni, I. Montvay, E. Scholz and L. Scorzato [qq+q Collaboration], Eur. Phys. J. C 31 (2003) 227; hep-lat/0307002; F. Farchioni, I. Montvay and E. Scholz [qq+q Collaboration], Eur. Phys. J. C 37 (2004) 197; hep-lat/0403014.

[16] L. Del Debbio, L. Giusti, M. Lüscher, R. Petronzio and N. Tantalo, JHEP 0602 (2006) 011; hep-lat/0512021.

[17] M. Göckeler et al., PoS LAT2006 (2006) 179; hep-lat/0610066.

[18] Ph. Boucaud et al. [ETM Collaboration], Phys. Lett. B 650 (2007) 304; hep-lat/0701012. 
[19] C. Morningstar and M. J. Peardon, Phys. Rev. D 69 (2004) 054501; hep-lat/0311018.

[20] R. Sommer, Nucl. Phys. B 411 (1994) 839; hep-lat/9310022.

[21] A. Morel, J. Phys. (France) 48 (1987) 1111.

[22] C. W. Bernard and M. F. L. Golterman, Phys. Rev. D 49 (1994) 486; hep-lat/9306005.

[23] S. R. Sharpe, Phys. Rev. D 56 (1997) 7052; [Erratum-ibid. D 62 (2000) 099901]; hep-lat/9707018.

[24] S. R. Sharpe and R. L. Singleton, Phys. Rev. D 58 (1998) 074501; hep-lat/9804028.

[25] W. J. Lee and S. R. Sharpe, Nucl. Phys. Proc. Suppl. 73 (1999) 240; hep-lat/9809026.

[26] G. Rupak and N. Shoresh, Phys. Rev. D 66 (2002) 054503; hep-lat/0201019.

[27] S. Aoki, Phys. Rev. D 68 (2003) 054508; hep-lat/0306027.

[28] O. Bär, G. Rupak and N. Shoresh, Phys. Rev. D 70 (2004) 034508; hep-lat/0306021.

[29] S. R. Sharpe and N. Shoresh, Phys. Rev. D 64 (2001) 114510; hep-lat/0108003.

[30] P. Weisz, Nucl. Phys. B 212 (1983) 1; P. Weisz and R. Wohlert, Nucl. Phys. B 236 (1984) 397 [Erratum-ibid. B 247 (1984) 544].

[31] P. de Forcrand and T. Takaishi, Nucl. Phys. Proc. Suppl. 53 (1997) 968; hep-lat/9608093.

[32] R. Frezzotti and K. Jansen, Phys. Lett. B 402 (1997) 328; hep-lat/9702016.

[33] I. Montvay and E. Scholz, Phys. Lett. B 623 (2005) 73; hep-lat/0506006; E. E. Scholz and I. Montvay, PoS LAT2006 (2006) 037; hep-lat/0609042.

[34] I. Montvay, Nucl. Phys. B 466 (1996) 259; hep-lat/9510042.

[35] R. G. Edwards, U. M. Heller and R. Narayanan, Nucl. Phys. B 535 (1998) 403; hep-lat/9802016.

[36] T. Kalkreuter and H. Simma, Comput. Phys. Commun. 93 (1996) 33; hep-lat/9507023.

[37] R. B. Lehoucq, D. C. Sorensen, and C. Yang, 1997, http: //www. caam.rice.edu/software/ARPACK/

[38] Y. Saad, "Numerical Methods for Large Eigenvalue Problems”, Manchester 1992.

[39] H. Neff, Nucl. Phys. Proc. Suppl. 106 (2002) 1055; hep-lat/0110076.

[40] A. M. Abdel-Rehim, R. Lewis and R. M. Woloshyn, Phys. Rev. D 71 (2005) 094505; hep-lat/0503007.

[41] M. Albanese et al. [APE Collaboration], Phys. Lett. B 192 (1987) 163.

[42] C. J. Morningstar and M. J. Peardon, Phys. Rev. D 60 (1999) 034509; hep-lat/9901004.

[43] J. Heitger, R. Sommer and H. Wittig [ALPHA Collaboration], Nucl. Phys. B 588 (2000) 377; hep-lat/0006026.

[44] J. Gasser and H. Leutwyler, Annals Phys. 158 (1984) 142; S. Dürr, Eur. Phys. J. C 29 (2003) 383; hep-lat/0208051.

[45] E. Witten, Nucl. Phys. B 156 (1979) 269; G. Veneziano, Nucl. Phys. B 159 (1979) 213.

[46] S. Dürr, Z. Fodor, C. Hoelbling and T. Kurth, JHEP 0704 (2007) 055; hep-lat/0612021.

[47] F. Sannino and M. Shifman, Phys. Rev. D 69 (2004) 125004; hep-th/0309252.

[48] F. Farchioni, I. Montvay, G. Münster, E. E. Scholz, T. Sudmann and J. Wuilloud, in preparation.

[49] K. Jansen, C. McNeile, I. Montvay, C. Richards, E. E. Scholz, C. Urbach and U. Wenger, PoS LATTICE2007 (2007) 036; arXiv:0709.4434 [hep-lat]. 